\title{
CURRENT TRENDS IN AMORPHOUS MAGNETISM
}

\section{J.M.D.Coey and D.H.Ryan}

Abstract. Recent research on some topics in amoxphous magnetism is reviewed and questions are posed which should be answered by future work. The topics are i) appearance of magnetism in binary alloys of the $3 \mathrm{~d}$ transition elements $\mathrm{Fe}$, Co and Ni with sp elements, ii) conflicting ground states of amorphous iron (ferromagnetic, asperomagnetic, paramagnetic), inferred by extrapolating different $\mathrm{Fe} M$ systems to $x=100$, iii) influence of hydrogen ${ }^{x}$ on the magnetic properties of metallic glasses, iv) random magnetic anisotropy and $v$ ) amorphous ionic compounds with purely antiferromagnetic interactions.

\section{INTRODUCTION}

The magnetic properties of amorphous materials have been studied rather intensively over the past ten years. With the development of methods of liquid and vapour quenching it has become possible to produce many metastable metallic alloys as well as a number of simple noncrystalline ionic compounds. These materials have extended our knowledge and improved our understanding of each of the two major categories of magnetic materials, metals and insulators. 1984 has seen the appearance of the first monographs in English on amorphous magnetism, one descriptive [1], the other theoretical [2], and substantial sections of other recent works on metallic glasses are devoted to their magnetic properties $[3,4]$. A book in German by Handrich and Kobe was published earlier [5] and there is a useful bibliography covering work up to 1980 [6]. Attention here is directed to some current trends in research in this field. Topics chosen are related to the magnetic moments and spin structures of amorphous metals and insulators. Each section ends with one or two questions which should be answered by future work.

\section{APPEARANCE OF 3d MOMENTS}

The use of vapour quenching to prepare simple binary alloys of a $3 d$ transition element with a non-magnetic element, $T_{100}$, which have non-crystalline structures over a wide range of composition has led to the prospect of examining systematically the condition for the appearance of $3 \mathrm{~d}$ magnetism. Following Rainford [7], it is useful to distinguish type $I$ onset of magnetism, where the $T$ atoms retain a magnetic moment even when they are rather dilute in $M$, and type II onset, where $T$ atoms are actually nonmagnetic when dilute in $M$. In the first case, magnetic order sets in at a critical concentration $x$ where the $T$ atoms are close enough for exchange interaetions to couple most of them together. If these interactions extend only to nearest-neighbours then the critical concentration is the site percolation threshold $x$ which is approximately $200 / \mathrm{N}$ in three dimensions, ${ }^{p}$ ( $N$ is the co-ordination number). A random dense-packed structure with $N=12$ therefore gives $x \simeq 17$. In the second case, the onset of magnetism occurs g $_{t}$ a concentration $x>x$ which depends sensitively on the number and type of nearest neighbours at the transition metal sites. The electronic configurations of the neighbouring atoms critically influence the width and spin polarization of the atomic $3 d$ levels, so that the onset of magnetism reflects not merely the geometry of the structure but also the nature of the elements concerned and the degree of hybridization and charge transfer involving 3d orbitals. The critical

Manuscript received ApriI 4, 1984.

The authors are at the Department of Pure \& Applied Physics, Trinity College, Dublin 2, Ireland. concentration in either case may be influenced by chemical short-range order.

Iron.

Much information is available on iron alloyed with an sp element from subgroups IIIA, IVA, VA, IIIB, IVB or VB of the periodic table[8-22]. Fe M alloys are normally ferxomagnets when the X X moment, but random non-collinear structures are occasionally found (e.g. when $M=Y)$. The average iron moment $\bar{\mu}$ for ferromagnetic compositions may be deduced from the spontaneous magnetization obtained from the magnetization curves at low temperature, assuming no moment resides on $M$. It is possible that a small antiparallel moment could be induced on IVA or VA subgroup metals, but this has yet to be demonstrated for an amorphous material. Alternatively, both the average iron moment and its probability distribution $\mathrm{P}(\mu)$ may be inferred from the hyperfine field distribution $P\left(B_{h f}\right)$ found from Mössbauer spectra. Proportionality between $\mu$ and $B$ has been established in a number of crystalline and $h f$ amorphous binary systems with slopes in the range $12.5-15.5 \mathrm{Tes} l a / \mu_{B}[23,24]$.

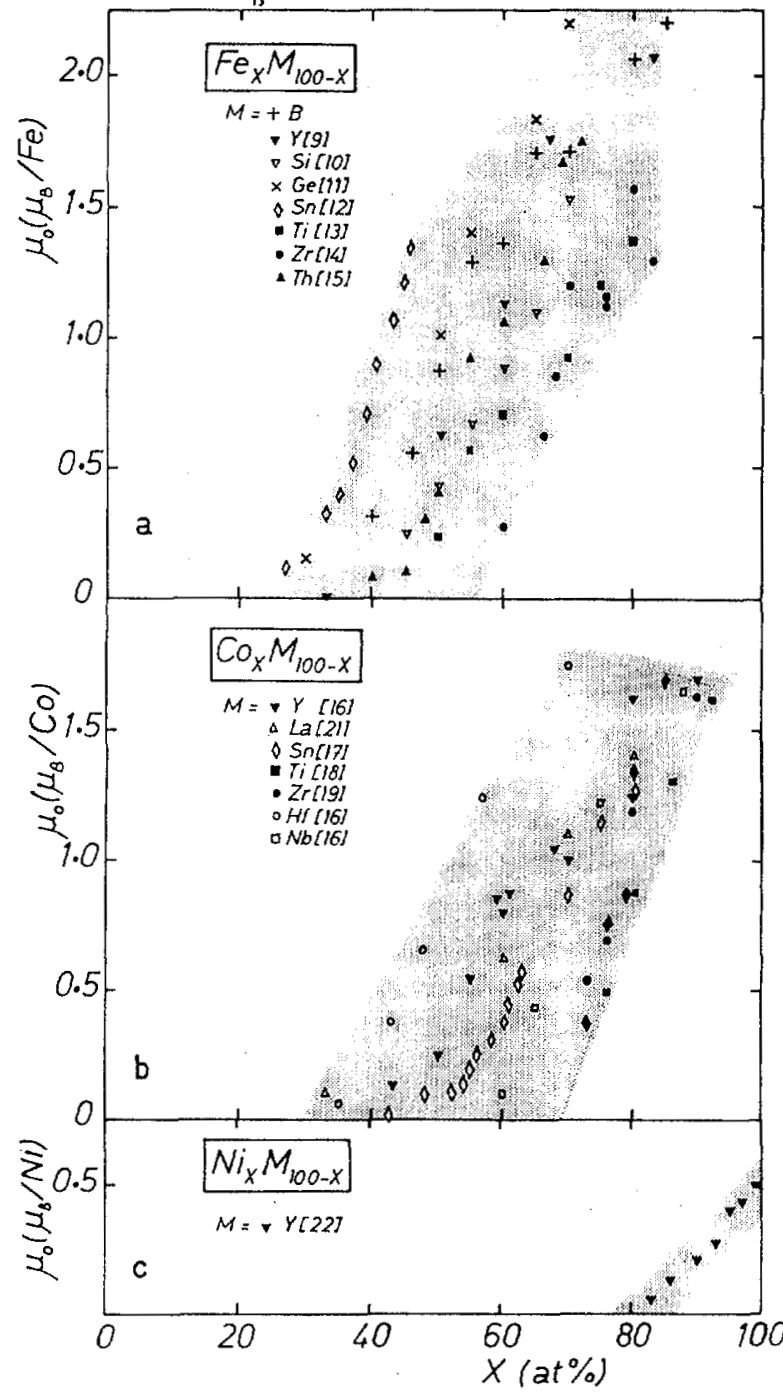

Fig. 1. Variation of the average transition-metal moment in amorphous binary alloys of (a) $\mathrm{Fe}$ (b) $\mathrm{Co}$ and (c) Ni with elements from subgroups IIIA,IVA, IIIB \& IVB 
A collection of results for $\overline{1}(x)$ for alloys of $F e$ with subgroup III and IV elements $P$ s shown in $\mathrm{fig}$. 1a. Mössbauer data, converted at $14 \mathrm{~T} / \mu$, allow the average iron atomic moment to be included even for systems with non-collinear structures. The critical concentration is in the range $30 \leqslant x \leqslant 55$, so that the appearance of magnetism is of type ${ }^{c} I I$. The influence of local environment on the appearance of an iron moment is directly reflected in the hyperfine field distribution $P\left(B_{f}\right)[8,25-29]$. Typically, when $x \geqslant x$ there is a broad peak centred near $4 \mathrm{~T}$ and another ${ }^{c}$ at higher fields. The first is usually attributed to iron in environments where it would not normally be magnetic, and the observed field may be a transferred hyperfine field due to conduction electron polarization or else it may reflect a weak spontaneous or induced moment on the iron. The high-field peak in the $P\left(B_{H}\right)$ distribution represents iron with a good spontaneougt moment. Coexistence of strongly magnetic and nonmagnetic iron (or iron with a very weak moment) has been demonstrated to extend over a range of concentration above $x$ in alloys with B[8], Si[25], $\mathrm{Ge}[26], \mathrm{Sn}[27]$ and $\mathrm{Y}[28]$, and in some cases the amount of nonmagnetic iron is proportional to the fraction of iron having fewer than 5 or 6 iron nearest-neighbours, assuming a simple model of random dense packing. It should be mentioned that procedures for generating $P\left(B_{f}\right)$ from Mössbauer spectra in the presence of an appreciable quadrupole interaction involve severe approximations, either treating the quadrupole-split central part of the spectrum as magnetic hyperfine splitting, or cutting it out and ignoring magnetic interactions entirely in the central part. The form of the $\mathrm{P}\left(\mathrm{B}_{\mathrm{ff}}\right)$ distribution below $2 \mathrm{~T}$ ( $\equiv 0.15 \mu_{\text {}}$ ) may be regarded whth scepticism, but the broad distribution of hyperfine fields above $x$ and the inhomogeneous nature of the appearance of $c$ magnetisn with coexistence of iron having a substantial moment with iron which is nonmagnetic (or weakly magnetic) is not in doubt. Evidence for chemical short range order has been seen in the relation between $B$ and the enthalpy of formation of $\mathrm{Fe}_{\mathrm{x}} \mathrm{M}_{100}$ alloys with $\mathrm{x} \sim 65$ [30].

The $\mathrm{x}$ influence of a11 $3 d$ transition metal impurities on the iron hyperfine field has been examined in the $\left(\mathrm{Fe}_{x} \mathrm{~T}_{100_{-}}\right) \mathrm{B}_{15} \mathrm{Si}_{13}$ system [31]. For $\mathrm{T}$ elements from Ti to $\mathrm{Xn}_{\mathrm{Mn}}$ there is a large effect, $\mathrm{dB} / \mathrm{dx}$ $\sim 0.5 \mathrm{~T}$, whereas from $\mathrm{Fe}$ to $\mathrm{Cu}$ the effect is very huch less (fig. 2). The particular effectiveness of $\mathrm{Cr}$ in destroying the iron moment is evident in

$\left(\mathrm{Fe} \mathrm{Cr}_{100}\right) \mathrm{B}_{20}$ alloys, for which $\mathrm{x}=60$ [29]. By contrast, there is no evidence of $a^{c}$ significant reduction in iron moment in $\mathrm{Fe}_{\mathrm{M}} \mathrm{M}_{100}$ when $\mathrm{M}$ is an element from group I ( $\mathrm{Ag}[32])$ or II $\mathrm{Mg}[30])$ but data on these systems is still very sparse. Only alloys of the type (Fe Ni, S $\mathrm{G}$ with $\mathrm{G}=\mathrm{B}, \mathrm{C}, \mathrm{Si} \ldots$ appear to

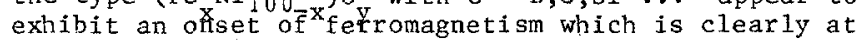
the percolation threshold $\mathrm{x} \sim 19[33,34]$ (type $\mathrm{I}$ ). A spin glass phase appears at ${ }^{C}$ greater dilution.

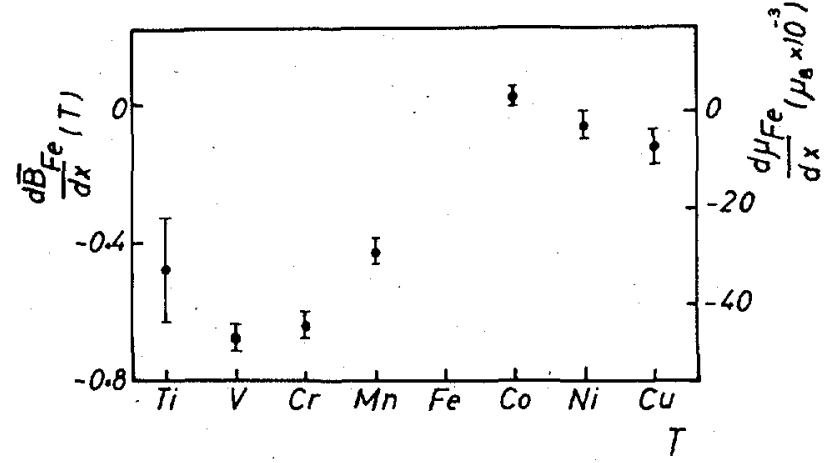

Fig. 2. Variation of ${ }^{57} \mathrm{Fe}$ hyperfine field and iron moment with transition metal impurities in ( Fe T) ${ }_{78} \mathrm{~B}_{12} \mathrm{Si}_{10}$ (afcer ref 31 ).

\section{Cobalt \& Nickel}

The variation of $3 \mathrm{~d}$ moment is shown for binary Co and $\mathrm{Ni}$ amorphous alloys in figures $\mathrm{Ib}$ and $\mathrm{IC}$. Some cobalt points were obtained from ferromagnetic resonance data, assuming a density obtained by interpolating the elemental liquid densities. The critical concentrations for the cobalt alloys fall in the range $40 \leqslant x \leqslant 70$, whereas for nickel $x \sim 80$. A very low critical concentration $x=41$ is found for $\mathrm{Ni}-\mathrm{Ag}[35]$, but for $\mathrm{Ni}-\mathrm{Mg} \mathrm{x}$ is $72[16]$.

The systematic variation of $x$ for binary alloys of $\mathrm{Fe}$, Co and $\mathrm{Ni}$ is summarized in Eable 1 . In general $\mathrm{x}_{(\mathrm{Ni})}>\mathrm{x}_{(\mathrm{Co})}>\mathrm{x}_{(\mathrm{Fe})}$ for a given $\mathrm{M}$, and the cfitical c8ncentratfon increases with the number of $\mathrm{sp}$ electrons on $M$ for a given $T$. Values of $x$ within each colum are fairly similar. These systematics are broadly in accord with the predictions of the simple slater-pauling mode1, which takes no account of atomic structure or nearest-neighbour environments. This model has recently been revived by Williams et al [36] with the concept of magnetic valence. Magnetic valence is defined as

$$
\mathrm{z}_{\mathrm{m}}=2 \mathrm{~N}_{\mathrm{d}}^{\uparrow}-\mathrm{Z}
$$

where $Z$ is the normal chemical valence (e.g. 3 for $Y$, 4 for Si, 8 for $\mathrm{Fe}$ ) and $\mathrm{N}^{\uparrow}$ is 5 for iron and elements to its right, which are supposed to have full spin-up $3 \mathrm{~d}$ bands, and $O$ for elements to its left. There is a further contribution from the $\mathrm{sp}$ band so that $\mathrm{N}^{\uparrow}=$ $\mathrm{N}_{\mathrm{d}} \uparrow+\mathrm{N}_{\mathrm{sp}}{ }^{\uparrow}$. The magnetic moment is then

$$
\mathrm{M}=z_{\mathrm{m}}+2 \mathrm{~N}_{\mathrm{sp}} \uparrow
$$

$z_{m}$ is to be averaged over the alloy composition, and $\mathrm{N}^{\mathrm{m}} \uparrow$ is taken as 0.3 . The predictions of the model are included in table 1 . It roughly reproduces the observed trends, although deviations from the assumed strong ferromagnetism (i.e. $\mathrm{N}_{3} \uparrow=5$ ) are evident in all iron-rich alioys. No more than a crude indication should be expected of a model that depends only on counting the number of electrons in the alloy.

\begin{tabular}{|c|c|c|c|c|c|c|c|c|c|c|c|}
\hline$I I I$ & & & & IV & & & & $V$ & & & \\
\hline & & co & & & $\mathrm{Fe}$ & $C_{0}$ & & & & co & \\
\hline$B$ & 38 & - & - & Si & 39 & - & - & $P$ & 45 & 68 & 75 \\
\hline$\gamma$ & 36 & 45 & 80 & $G e$ & 40 & - & - & $S b$ & 43 & - & - \\
\hline $\mathrm{La}$ & - & 42 & - & Sn & 35 & 51 & - & Nb & 56 & 59 & - \\
\hline & & & & $T i$ & 43 & 69 & - & $\mathrm{Ta}$ & $n$ & - & - \\
\hline & & & & $Z r$ & & 64 & 85 & & & & \\
\hline & & & & Hf & & 37 & - & & & & \\
\hline & & & & $T h$ & 43 & - & - & & & & \\
\hline$e q$. & & 60 & 80 & & 57 & 68 & 85 & & 63 & 73 & 88 \\
\hline
\end{tabular}

Taule 1. Critical concentrations for Fe, Co \& Ni a'lloys

Although we have defined $x$ by extrapolating the rapidly-falling sections of the $C_{T}, \vec{\mu}_{z}$ or $B_{\text {f }}$ curves to zero, the magnetic properties fave only been examined at low temperatures in the vicinity of $x$ for a few systems including Fe-Sn[12] and Ni-Y [37]. \& spin glass phase found there for $x<x$, probably involves only the fraction of $T$ atoms $\xi i t u a t e d$ in environments favourable for moment formation, which interact via weak long-range interactions.

The challenge is now to explain the appearance of $3 d$ magnetism on the basis of electronic structure calculations for realistic models of non-crystalline binary alloys. Two questions which must be answered by further experimental work are: When do $3 \mathrm{~d}$ elements retain their moments as impurities in a nonmagnetic amorphous matrix? Does a spin glass phase just below $x$ inevitably accompany the onset of magnetism in aforphous binary alloys? 


\section{THE PUZZLE OF AMORPHOUS IRON}

There are no modern reports on magnetic properties of we1l-authenticated examples of pure amorphous iron. on the contrary, evidence indicates that the pure metal cannot be stabilized, even at $4 \mathrm{~K}$ [38]. Samples originally reported as being amorphous iron have subsequently turned out to contain significant, uncontrolled amounts of impurity such as $C$ [39].

The most promising approach to the problem has been to extrapolate the properties of series of iron-rich $\mathrm{Fe} \mathrm{M}_{100}$ alloys to $x=100$. Systems that have been investigated In some detail include

$$
\begin{aligned}
& \mathrm{Fe}-\mathrm{B} \text { up to } \mathrm{x}=90[8,11,40,41] \\
& \mathrm{Fe}-\mathrm{Y} \text { up to } \mathrm{x}=88[9,28]
\end{aligned}
$$$$
\mathrm{Fe}-\mathrm{Zr} \text { up to } \mathrm{x}=93[12,24,42,43]
$$

The magnetic ground state of these alloys has been characterized from Mössbauer data and magnetization curves at low temperatures. From the former, the distribution of atomic moment $P(\mu)$ and its average value $\bar{\mu}$ may be inferred, whereas the latter gives the average component of the moment in the direction of the field $\bar{\mu}$. If $\bar{\mu} \simeq \bar{\mu}$, the alloy is ferromagnetic but if $\vec{\mu}$ is signiticantiy less than $\vec{\mu}_{0}$, then the structure must be noncollinear.

Some ferromagnetic amorphous $\mathrm{Fe} \mathrm{M}_{100-\mathrm{O}}$ systems show a fall in Curie temperature as $x \rightarrow 100^{-x}$ ( $f$ ig. 3a). The explanation may be collapse of the iron moment or weakening of the ferromagnetic exchange interaction or both. A reduction in iron magnetization $\bar{\mu}$ in the iron-rich limit may also reflect a reduction in the iron moment or else the onset of a random noncollinear, asperomagnetic spin structure (fig. 5) because of a shift in the exchange distribution $P(\mathscr{f})$ to encompass a substantial minority of antiferromagnetic

interactions. Data in fig. 3 on the three alloys systems cited above illustrate these effects. a-Fe $B_{100}$ shows no sign of any reduction in iron moment with increasing $x$, but it does show a decrease in $T$ which reflects a weakening of the net ferromagnetic ${ }^{c}$ exchange. This may reflect the presence of some antiferromagnetic interactions, but they are

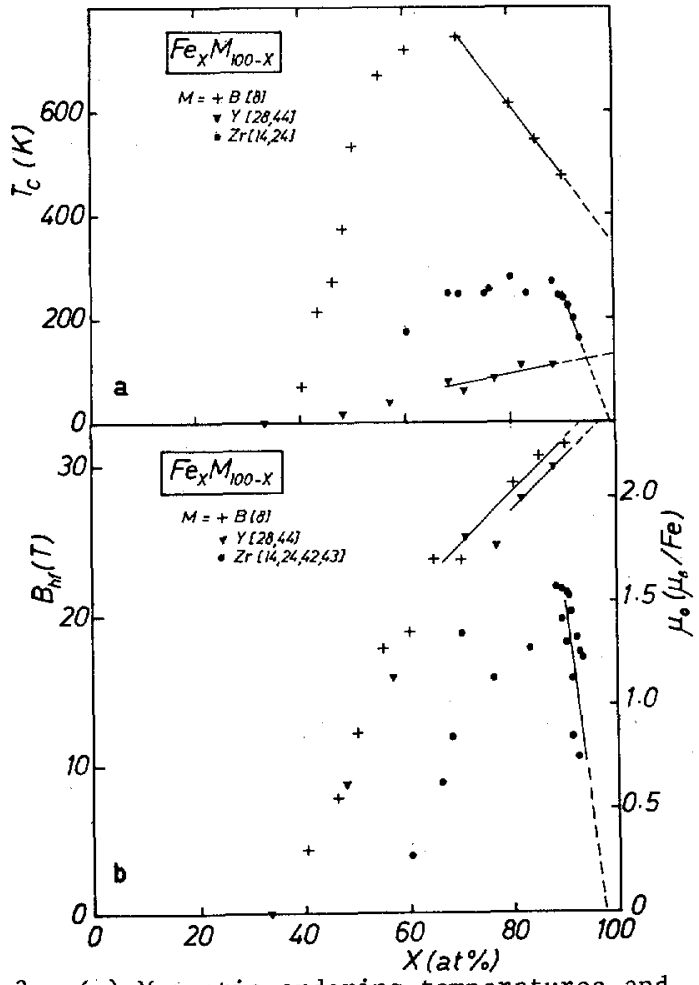

Fig. 3. (a) Magnetic ordering temperatures and (b) average atomic moment for amorphous iron alloys with extrapolations to $x=100$. insufficient to destroy the collinear ground state. The extrapolated anorphous iron is therefore ferromagnetic with a moment $\bar{\mu}$ of about $2.3 \mu_{B}$ and a Curie temperature close to ro8m temperature. ${ }^{B}$ We will call this $\alpha$-amorphous iron.

A second form, B-amorphous iron, may be inferred from Fe $\mathrm{Y}$ - Here the average atomic moment $\bar{\mu}$ behaves ${ }^{x}$ similarly to that of Fe-B. However the alloys are not ferromagnetic at any composition, but are asperomagnets instead. A fraction of the atomic moment can be easily saturated in an applied field, but no laboratory field is enough to align the moments completely [44]. The random noncollinear structure is attributed to a substantial minority of antiferromagnetic interactions which destabilize the ferromagnetic state. The moment $\bar{\mu}$ extrapolated to $\mathrm{x}=100$ is about $2 \mu$, but the spin fleezing temperature is only $130 \mathrm{~K}$. Nón other Fe-M systems with similar properties have been described, but Fe-Lu and $\mathrm{Fe}-\mathrm{Sc}$ are likely candidates.

a-Fe $2 \mathrm{r}_{100}, \mathrm{O}, 1 \mathrm{ke} \mathrm{a}-\mathrm{Fe} \mathrm{B}_{100_{-x}}$, has the Curie temperature passing through a maximum near $x=80$. However, as $x \rightarrow 100$ both $T$ and the iron moment decline sharp1y. Extrapolation Eo $x=100$ indicates that the moment would have disappeared entirely in pure amorphous iron which would then have a paramagnetic ground state with enhanced susceptibility. Fe-Hf appears to behave similarly[42]. We may call this non-magnetic variety $\gamma$-amorphous iron. As a general tendency, it is observed that amorphous iron alloys with a substantially weakened iron moment or a reduced Curie temperature tend to show the invar effect [45]. This is true of iron-rich Fe-Zr [43], and Fe-B [45] as well as several alloys with $x$ just greater than the critical concentration for the appearance of magnetism $[47]$.

Table 2. Magnetic properties of amoxphous iron at $O K$ extrapolated from amorphous binary Fe-M alloys

$\begin{array}{cccccccc}M & \begin{array}{c}\mathrm{B} f \\ \mathrm{hf}\end{array} & \bar{\mu}_{\mathrm{o}} & \vec{\mu}_{z} & \text { Magnetic } & T_{\dot{0}} & \text { Form } & \text { Ref. } \\ & (\mathrm{T}) & \left(\mu_{\mathrm{B}}\right) & \left(\mu_{\mathrm{B}}\right) & \text { State } & (\mathrm{K}) & & \\ \mathrm{B} & 32.0 & 2.3 & 2.3 & \text { Ferromagnetic } & 300 & \alpha & {[8]} \\ \mathrm{Y} & 32.2 & 2.3 & 1.6 & \text { Asperamagnetic } & 130 & \beta & {[28,44]} \\ \mathrm{Zr} & 0 & 0 & 0 & \text { Paramagnetic } & - & \gamma & {[24]}\end{array}$

It is, of course, conceivable that the trends visible in $\mathrm{fig} .3 \mathrm{might}$ reverse themselves and come together at even higher iron content where it has not yet been possible to prepare the alloys in their amorphous form. But the data, as of now, suggest that there may be three different varieties of amorphous iron, one nonmagnetic, the others with moments of around $2 \mu / \mathrm{Fe}$ arranged either in a ferromagnetic or in an asperomagnetic configuration. There is no reason to believe that there must be a unique form of noncrystalline iron: amorphous polymorphism is already known for metallic glasses [48]. In the crystalline state, bcc and hcp iron have quite different magnetic properties, and the behaviour inferred for fcc iron depends on the alloy system from which one extrapolates [49]. Questions which now arise are: What is the structural basis of the three magnetic variants? Can transitions between them occur as a function of temperature or pressure? [51]

\section{HYDROGEN}

Studies of the influence of hydrogen on the magnetic properties of metallic glasses have begun recently $[24,43,50-53]$. Alloys may be loaded by gas. pressure, electrolysis or proton implantation. Certain compositions such as $\mathrm{Zr}_{75} \mathrm{Rh}{ }_{25}$ may be prepared directly in the amorphous hydrided form by simply exposing the crystalline alloy to gas pressure [55]. Others, 
particularly in the Ni-Zr system, are attractive for hydrogen storage applications since they suffer less damage on absorption-desorbtion cycling than do crystalline alloys [56]. Usually it is necessary to use hydrogen pressures of order 1MPa (10 bars) and heat the sample somewhat to load them, but the need for elevated pressures and temperatures may be overcome by evaporating a thin layer of palladium onto the sample surface to act as an $\mathrm{H}_{2}$ dissociation catalyst [54].

The electrolytic method [24] supplies a stream of dissociated hydrogen atoms directly to the surface of the sample, which is the cathode of an electrolytic cell containing a non-acid electrolyte such as $\mathrm{K}_{2} \mathrm{CO}_{3}$ and a Pt anode. The method is applicable so long as the cathode does not fall apart, which means that very low currents sometimes have to be used.

The maximum hydrogen loading attainable is roughly three hydrogen atoms per early transition metal (e+g. $Y, Z r)$ or rare-earth. This fact indicates that an anionic picture is appropriate, whereby more than one electron resides in the vicinity of the proton. In crystals the density of lower-lying bonding states is strongly modifled by hydrogen, and split-off metal-hydrogen bands appear 5-7 eV below the Fermi leve1 [57]. Differential scanning calorimetry shows that the binding energy is of order $1 \mathrm{eV} / \mathrm{H}[51]$, and that hydrogen desorbtion begins around $400 \mathrm{~K}$ for $\mathrm{Ni}_{36} \mathrm{Zr}_{64}$ [56] and $\mathrm{Fe}_{91} \mathrm{Zr}_{9}[24]$, and around $700 \mathrm{~K}$ for $\mathrm{Fe}_{4} \mathrm{Y}_{60}[24]$. A sample of hydrogenated $\mathrm{Fe}_{88} \mathrm{Y}_{12}$ has shown practically no change in hydrogen content over a period of two years whereas iron-rich $\mathrm{Fe}-\mathrm{Zr}$ alloys lose their charge in a matter of weeks [24].

Magnetic properties of amorphous alloys change on hydrogenation because of the substantial increase in volume which may reach $20 \%$ in some cases, and because of the change in electronic structure. The volume effect is most important for iron-rich Fe-M alloys where $M$ is a rare-earth or yttrium. The Fe-Fe direct exchange is a sensitive function of nearest-neighbour distance, and the downturn in Curie temperature and non-collinear spin structures have both been attributed to broad $P(\mathcal{F})$ distributions which include negative or only weak positive interactions corresponding to the shortest $\mathrm{Fe}-\mathrm{Fe}$ bonds. Hydrogen should have a dramatic effect on the magnetic properties of these alloys and indeed it does. a-Fe $\mathrm{Y}_{100}$ with $75 \leqslant x \leqslant 88$ change from asperonagnets with a spin freezing temperature of $120 \mathrm{~K}$ to good soft ferromagnets with $T \cong 400 \mathrm{~K}$. There is little change in $\bar{\mu}$, the average ${ }^{c}$ iron moment. However, the Curie temperature of ferrimagnetic a-Fe Gdioo which is approximately $450 \mathrm{~K}$ regardless of composition for $60<x<90$, decreases by $50 \mathrm{k}$ for an alloy with $\mathrm{x}=$ 67. The compensation temperature is also reduced by bydrogen [54]. These effects reflect a substantial shift of Gd-Gd exchange towards antiferromagnétic values which may be attributed to a change in Gd conduction electron density.

Another effect is found in iron-rich $\mathrm{Fe}-\mathrm{Zr}$ alloys which have a reduced iron moment (fig. 3). The moment is restored to its full value of $\sim 2 \mu$ on hydrogenation, and there is a corresponding ${ }^{B}$ increase in Curie temperature. The spontaneous volume

magnetostriction and invar effect are not affected, hut coercivity which appears in the liquid helium range for these materials is reduced by a factor of 10 [43]. Comparison of the volume dependence of the Curie temperature on hydrogenation with the effect of pressure, assuming a bulk modulus measured on similar compositions, shows a quite different slope in the two cases (fig. 4), indicating that there is an influence of hydrogen on the electronic structure beyond the normal volume effect. A similar conclusion is reached from isomer shift and ESCA measurements. In summary, the effect of hydrogen in iron-rich alloys appears to be to convert $\beta$ or $\gamma$ amorphous iron to the $\alpha$ modification. In iron-poor alloys with rare-earths where only the rare-earth is magnetic, hydrogen has the opposite effect, depressing the Curie temperature and precipitating spin glass behaviour $[53,58]$.

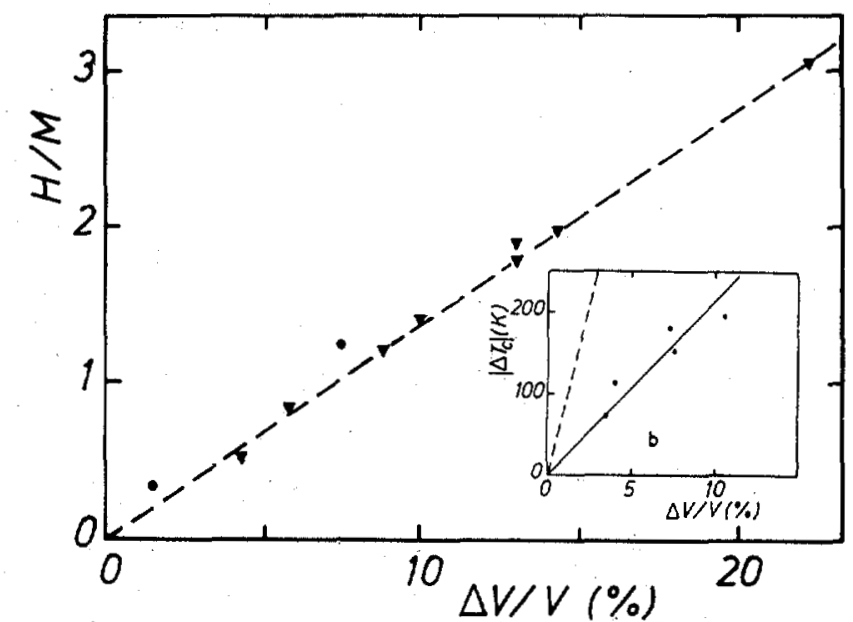

Fig. 4. Hydrogen content as a function of volume change for $-\mathrm{Fe}-\mathrm{Zr} \vee \mathrm{Fe}-\mathrm{Y}$ anorphous alloys. The insert shows the change in Curie temperature with volume increase on hydrogenation for iron-rich $\mathrm{Fe}-\mathrm{Zr}(\bullet)$ and volume decrease under pressure $\cdots-[78]$

Questions to be answered by future work in this axea are: Is it possible to make use of hydrogen to tailor the magnetic properties of alloys for specific applications? Can the influence of volume and electronic structure changes an magnetic properties be unraveled?

\section{RANDOM SINGLE-ION ANISOTROPY}

The electrostatic field acting on the $4 \mathrm{f}$ shell of rare-earth atoms in amorphous alloys creates local, random single-ion anisotropy which leads to random noncollinear magnetic structures. To describe the interaction of a single ion having total angular momentum quantum number $J$ with the electrostatic field at its site, the following Hamiltonian is used

$$
\mathcal{H}_{\text {ef }}=\sum_{\mathrm{B}}^{\mathrm{m}} \hat{0}_{n}^{\mathrm{m}}
$$

where $n=0,2,4,6$ and $|m| \leqslant n$. Coefficients $B m$ in the expansion are the local electrostatic field parameters and $\hat{O} m$ are the Stevens operators. Most of these terms are absent by symmetry in crystals but 28 of them are needed to describe the interaction in the general case. The problem evidently calls for radical simplification. By far the most popular course has been to consider only second order terms. With a suitable choice of axes, only the terms in $\hat{0}_{2}{ }^{0}$ and $\hat{0}_{2}{ }^{2}$ remain. If the of $f$-diagonal term is also neglecetd, the Hamiltonian reduces to $\mathrm{B}_{2} \mathrm{O}_{2} \hat{\mathrm{O}}^{\mathrm{O}}=\mathrm{B}_{2}{ }^{0}\left(3 \vec{J}{ }^{2}-J(J+1)\right)$. An equivalent form is $-D \vec{J}, 2$, where the easiest direction $z$ varies from site tzisite ( $D$ is positive). In the random axial anisotropy model, introduced by Harris, Plishke and 7.ukermann in 1973 [59] $z$ is assumed to vary in a randon, isotropic manner from site to site. The tamiltonian is

$$
\sum_{i>j} f_{i j} \vec{J}_{i} \cdot \vec{J}_{j}-\left[0 \vec{J}_{z i}^{2}\right.
$$

Although highly simplified, this model provides a good starting point for understanding magnetic properties of rare-earth amorphous alloys whose random, noncollinear structures are dominated by single-ion anisotropy [60]. These structures may be partially characterised by neutron diffraction [61]. There is a large literature on the random axial anisotropy model [60], second only to that on the random exchange model. 
Even very weak anisotropy $(\alpha=D / Z<<1$, where $Z$ is the coordination number) will theoretically destabilize the ferromagnetic state in favour of one where the direction of average local magnetization wanders continuously throughout the sample. (It has been suggested, however, that the susceptibility may still diverge [62]). In the limit $\alpha<<1$, this state would be practically indistinguishable from a soft ferromagnet, but as $\alpha$ increases, there is an abrupt changeover to a strongly-pinned regime when $\alpha>0.4$. Each spin is then close to its local easy direction. Treating the model in the molecular field approximation with a unique $z$-direction for the molecular field leads to the asperomagnetic state illustrated in fig. 5 with an average moment $\bar{\mu}_{z} \geqslant 1 / 2 \bar{\mu}$. However, more realistic approaches involving computer simulation or length-scale arguments indicate that a state with no net moment is marginally lower in energy, and that the local $z$-axis wanders over distances of order several interatomic spacings, a tiny 'asperomagnetic domain' whose size depends on the range of the ferromagnetic exchange interaction. Nevertheless, an applied field will stabilize the bulk asperomagnetic state of $\mathrm{fig} .5$, thereby explaining why in low temperature magnetization curves of many rare-earth amorphous alloys a relatively small field will induce about half the moment, but an enormous one is needed to approach saturation (as $\left.1 / B^{2}\right)$. Besides magnetization, the model has also had some success in explaining the hysteresis,

low-temperature specific heat and magnetoresistance of rare-earth glasses [60].

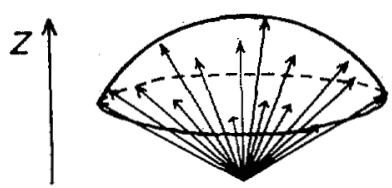

Fig. 5. An asperomagnetic spin structure.

The next step has been to include the other second order term $\mathrm{B}_{2}{ }^{2 \hat{O}_{2}}{ }^{2}$ which lifts the degeneracy for non-Kramers ions. Agreement with measured magnetization and susceptibility of dilute alloys $\mathrm{Pr}^{3+}$ is improved thereby [63]. Besides choosing randomly oriented coordinate axes at each site, $\mathrm{B}_{2}{ }^{0}$ and $\mathrm{B}_{2}{ }^{2}$ should be allowed to take a distribution of values. principle, these second order electrostatic field parameters and their distributions are obtainable directly from measurements of nuclear quadrupole interactions by $\mathrm{nmr}$ or Mössbauer spectroscopy since $\mathrm{B}_{2}{ }^{\mathrm{O}}$ is proportional to $\mathrm{V}$, the principal component of the electric field gradient, and $\mathrm{B}_{2}{ }^{2} / \mathrm{B}_{2}{ }^{\mathrm{D}}=n$, the asymmetry parameter[64]. In practice, it has proved difficult to obtain much more than average values of the two parameters because of the limited resolution of the experimental probes [65], although the new $174 \mathrm{Yb}$ resonance is promising[66]. The distribution of splitting of the ground and excited states has been probed directly by inelastic neutron scattering [67]. The distributions of the parameters, $V$ and $\eta$ have been calculated for simple structural hodels [68]. By contrast, there has been little attempt to examine anything other than the second-order electrostatic field in amorphous solids, although the approximation is inadequate to explain the magnetization curves of some dilute light rare-earth alloys and heavy rare-earth glasses [60]. While it is obviously impossible to take more than a few terms into account, it has been suggested that a field of random hexagonal symmetry [69], or one with a few terms to represent quasicrystalline atomic environments randomly oriented [60] may be better. The question to be resolved here is: Can any alternative simplification of the electrostatic field Hamiltonian be as successful as the random axial anisotropy model in explaining the magnetic properties of rare-earth alloys?

\section{AMORPHOUS MAGNETIC INSULATORS}

Ferrites and related non-metallic transition-metal compounds have been the objects of a great deal of research in magnetism. production of these materials for applications is comparable in value to that of ferromagnetic metals and alloys. Yet there is no research activity on amorphous magnetic insulators to compare with that on amorphous metallic alioys. Why not? The answer lies simply in the sign of the exchange. With few exceptions, like $a-\mathrm{FeF}_{2}[70]$ or nonstoichiometric a-FeGaO ${ }_{3}[71]$ where the exchange is positive (ferromagnetic) because of a special $\mathrm{Fe}^{2+}-\mathrm{F}-\mathrm{Fe} e^{2+}$ bond angle or $\mathrm{Fe}^{2+\rightarrow} \mathrm{Fe}^{3+}$ electron hopping, the superexchange interactions in $3 \mathrm{~d}$ compounds are usually negative (antiferromagnetic). Crystalline ferrimagnets owe their useful magnetic properties to crystal structures in which the cation sites can be split into two (or more) unequal sublattices, so that negative intersublattice interactions lead to a net moment. This special topology appears in practice to be destroyed in amorphous oxides and fluorides, although there is no geometrical reason why that should necessarily be so. Fig. 6 a shows a noncrystalline network in which every antiferromagnetic $0^{-x}$ interaction could be satisfied, leading to a large net

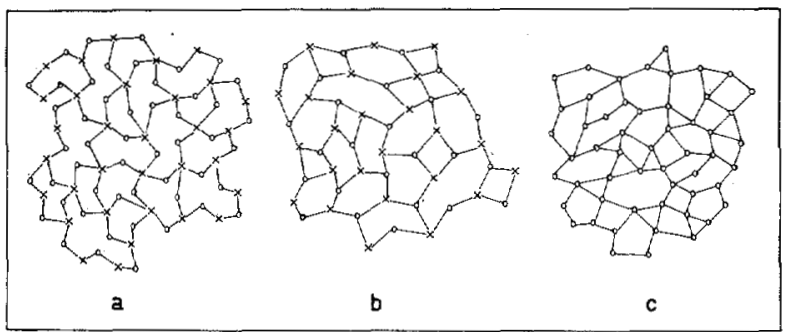

:ig. 6. Two dimensional noncrystalline networks.

moment. However the only amorphous ferrimagnets known are metallic Gd-T alloys ( $T=F e$, Co or Ni) where the two subnetworks are defined chemically rather than topologically, and ferrimagnetism is a product of positive $T-T$ interactions and weaker, negative $G d-T$ coupling. Turning to the special case of amorphous antiferromagnets, it is also possible to imagine a noncrystaline network composed of even-membered rings (figure 6b) which can be divided into two equal subnetworks in such a way that negative o-x interactions can all be simultaneously satisfied. Such a material should order as a collinear antiferromagnet. However, noncrystalline $3 \mathrm{~d}$ ionic compounds that have been examined to date [72] turn out to have their antiferromagnetic interactions frustrated by topological disorder of the type indicated in figure $6 c$ - odd-membered rings are distributed at random among the even-membered ones. In consequence, two subnetworks cannot be defined and a random noncollinear spin structure energes as a compromise ground state. This speromagnetic order may include antiparallel net nearest-neighbour correlations, but they fall of $f$ very quickly with distance [73].

The main consequence of frustrating the antiferromagnetic superexchange interactions is that the spin freezing temperature $T$ in noncrystalline compounds is reduced by an ordef of magnitude or more relative to the Néel temperature that would be found for an unfrustrated crystal (or an amorphous solid like fig. 6b). T does not exceed $150 \mathrm{~K}$ for $\mathrm{Fe}^{3+}$ compounds, and is considerably lower for $\mathrm{Mn}^{2+}, \mathrm{Co}^{2+}$ or the rare earth ions [72]. Magnetic properties around and below $T$ broadiy resemble those of the canonical spin glasses, except that light influences the magnetic relaxation [74]. Deviations from Curie-Weiss susceptibility $x=C /(T+\theta)$ usually set in well above $\tau_{f}$, with a reduced constant $C$, which reflects strong aftiparallel correlations of neighbouring spins. 
Table 3. Magnetic properties of crystalline and amorphous ferric compounds

$$
\begin{array}{lll}
T_{0} & \theta_{p} \\
(\mathrm{~K}) & (\mathrm{K}) & \\
& &
\end{array}
$$

$\begin{array}{llrrl} & \text { (crystalline) } & 363 & -610 & \text { Ant1ferromagnet } \\ & \text { (noncrystalline) } & 29 & -485 & \text { Speromagnet [76] } \\ & & & & \\ \mathrm{Y}_{3} \mathrm{Fe}_{5} \mathrm{O}_{12} & \text { (crystalline) } & 560 & -1100 & \text { Ferromagnet } \\ & \text { (noncrystalline) } & 40 & -500 & \text { Speromagnet [77] }\end{array}$

a-FeF 3 is a particularly simple example of a noncrystalline ionic compound whose structure can be modelled by an octahedral random network containing 3-,4- and 5-membered iron rings [75]. The crystal has only 4- membered rings, and the drastic differences in magnetic properties between crystalline and amorphous forms of this compound, and the well-known ferrimagnet $\mathrm{Y}_{3} \mathrm{Fe}_{5} \mathrm{O}_{12}$ are summarized on table 3 . It is worthwhile concentrating on these materials for which plausible structural models can be constructed rather than on complex silicate or other glasses if progress is to be made in relating structure to magnetic properties. Two questions which future research may answer are: Is it possible to devise materials with structures like those in fig. 6a) or b) which would be true amorphous ferrimagnets or anti Eerromagnets? Can spin freezing ever occur in systems with antiferromagnetic interactions and $\mathrm{s}<3 / 2$ ?

Acknowledgement: This work was supported, in part, by the CEC, contract No. SUM-041-EIR.

\section{REFERENCES}

[1] K. Moorjant and J.M.D. Coey, 'Magnetic G1asses' Elsevier, Amsterdam 1984.

[2] T. Kaneyosh1 'Amorphous Magnetism' CRC Press Boca Raton, Florida 1984.

[3] R. Hasegawa (editor) 'Glassy Metals: Magnetic, Chemfcal and Structural Propertfes' CRC Press Boca Raton, Florida 1983.

[4] F.E. Luborsky (ed1tor) 'Amorphous Metallic Alloys', Butterworths, London 1983.

[5] K. Handrich and S. Kobe 'Amorphe Ferro- und Ferrimagnetika' Physik-Verlag, Weinhe1m DDR 1980.

[6] A.R. Ferchmin and S. Kobe, 'Amorphous Magnetism and Metallic Magnetic Materials Digest' North Holland, Amsterdam 1983.

[7] B.D. Rainford and S.K. Burke, J. App1. Phys. 537660 (1982)

[8] C.L. Chien \& K.M. Unruh, Nucl. Inst. \&eth. 199 193 (1982)

[9] J. Chappert, R. Arrese-Boggtano and J.M.D. Coey, J. Magn. Magn. Mater. Z 175 (1978).

[10] G. Marchal, P.L. Mangin, M. Pfecuch, C. Janot and J. Hubsch, J. Phys. F7 LI65 (1977).

[11] K.H.J. Buschow \& P.G. v Engen, J. App1. Phys. 523557 (1981)

[12] M. Pfecuch, C. Janot, G. Marchal and M. Vergnat, Phys. Rev. B28 (1983).

[13] S.H. L1 ou and C.L. Chien, R. Sumiyama, Y. Hashimoto, T: Yoshitake and Y. Nakamura, J. Magn. Magn. Mater. 31-34 1495 (1983), J. Appl. Phys. 551820 (1984).

[14] K.H.J. Buschow \& P.H. Smit, J. Magn. Magn. Mat. 2385 (1981)

[15] K.H.J. Buschow and A.M. van der Kraan, Phys. Stat. Sol. (a) 53665 (1979)

[16] K.H.J. Buschow J. App1. Phys. 537715 (1982).

[17] G. MarchaI, D. Teirlinck, P. Mangin, C. Janot, and J. Physique 41 C8-662 (1980).

[18] J.A. Aboaf and E. Klokholm, J. Appl. Phys. 521844 (1981).

[19] R. Krishnan, M. Tayhouni, M. Tessiey and A. Gangulee, J. App1. Phys. 532243 (1982).

[20] M. Naoe, M. Kodaiya, Y. Hosht and S. Yamanaka, IEEE Trans. Mag. 173026 (1981).

[21] N. Heiman and N. Kazama, Phys. Rev. B17 2215 (1978).

[22] D. Gignoux, D. Givord and A. Liénard, J. Appl. Phys. 532321 (1982).

[23] P. Pannisad, J. Durand and J.I. Budnick, Mucl. Inst, and Meth. 199 99 (1982).

[24] J.M.D. Coey, D.H. Ryan and Yu Boliang, J. Appl. Phys. 55 1800 (1984).

[25] G. Marcha1, P. Mangin \& C. Janot, Solid State Comtun, 18739 (1976).

[26] O. Massenet, H. Daver, V.D. Nguyen and J.P. Rebouillat, J. Phys. F9 1687 (1979).

[27] B. Rodmacq, M. Plecuch, C. Janot, G. Marchal and P. Mangin, Phys. Rev. B21 1911 (1980).

[28] J. Chappert, J.M.D. Coey, A. Liénard and J.P. Rebouillat, J.
Phys. F11 2727

[29] Yu Boliang, J.M.D. Coey, M. Olivier and J.O. Ström-01sen, J. App1. Phys. 55 1748(1984).

[30] A.M. v. d. Kraan \& K.H.J. Buschow, Phys. Rev. B25 3311 (1982).

[31] T. Kemeny; B. Fogarassy, I. Vincze, I.W. Donald, M.J. Remus and H.A. Davies. Proceedings of 4 th Conference on Rapidly Quenched Metals, Sendai (1982).

[32] C.L. Chien and K.M. Unruh, Phys. Rev. B28 1214 (1983).

[33] J. Durand in "Glassy Metals, Magnetic Chemical and Structural \& Properties" CRC Press Boca Raton Florida (1983) p.109

[34] J.A. Geohegan \& S.M. Bhagat, J.Magn.Magn.Mat. 25143 (1981)

[35] J.J. Hauser, Phys. Rev. B12 5160 (1975).

[36] A.R. Williams, V.L. Moruzzi and A.P. Malozemoff (in press).

[37] A. Liénard, J.P. Rebouillat, P. Garoche and J.J. Veyssie, J. Physique 41 C8-658 (1980).

[38] M.R. Bennett and J.G. Wright, Phys. Stat. Sol. (a) 13135 (1972).

[39] S. Bjarman, R. Kamal and R. Wappling, J. Magn. Magn. Mater. 15-18 1389 (1980); S. Bjarman and R. Wappling, Report UU1P-1047 University of Uppsala, Institute of Physics 1981.

[40] R. Hasegawa and R. Ray, J. App1. Phys. 49, 4174 (1978).

[41] C.L. Ch1en, D. Musser, E.M. Gyorgy R.C. Sherwood, H.S. Chen, F.E. Luborsky and J.L. Walter, Phys. Rev. B20 283 (1979).

[42] T. Masumoto, S. Ohnuma, K. Shirakawa, M. Nose and K. Kobayashi, J. Physique. $41 \mathrm{C}(8-686$ (1980).

[43] H. Eujimori, K. Nakanishi, H. Hiroyoshi and N.S. Kazama, J. Appl. Phys, 53, 7792 (1982).

[44] J.M.D. Coey, D. Glvord, A. Liénard and J.P. Rebouillat, J. Phys., F11, 2707, (1981).

[45] K. Pukamichi, T. Masumoto and M. Kikuchi, IFEE Trans. Mag. MAG 151404 (1979).

[45] K. Fukamichi in ref. 4, p. 317.

[47] K. Fukamich1, K. Shirakawa, T. Kaneko and T. Masumoto, J. Appl. Phys. 53, 2246 (1982).

[48] B.W. Corb, R.C. O'Handley, J. Megusar and N.J. Grant, Phys. Rev. Lett. 51 1386 (1983).

[49] T. Kemeny, F.J. Litterst, I. Vincze and R. Wappling, J. Phys, F13 L37 (1983).

[50] J.M.D. Coey, D.H. Ryan, D. Gígnoux, A. Liénard and J.P. Rebouillat, J. Appl. Phys. 537804 (1982).

[51] Yu Boliang, D.H. Ryan, J.M.D. Coey, Z. Altounian, J.O. Ström-01sen and F. Razavi, J. Phys. F13 L217 (1983).

(52] B.S. Berry and W.C. Pritchet, J. App1. Phys. 521865 (1981).

[53] C.G. Robbins, Z.D. Chen, J.G. Zhao, M.J. O'Shea and D.J. Sellmyer, J. Appl. Phys. 537798 (1982); D.J. Sellmyer, C.G. Robbins and M.J. O'Shea, J.Noncryst. Solids (in press).

[54] J.H. Schelleng, D.W. Forrester, P. Lubitz and C. Vittoria, J. Appi. Phys. 55805 (1984).

[55] X.L. Yeh, K. Samwer and W.L. Johnson, Appl. Phys. Lett. 47, 242 (1983).

[56] F.H.M. Spit, J.W. Drijver, W.C. Trukenburg and S. Redelaar, J. Physique, 41 c8-890 (1980).

[57] J.W. Weaver, D.J. Peterman and D.T. Peterson in 'glectronic Structure and Properties of Hydrogen in Metals' Plenum. New York 1982 p.207.

[58] D.J. Sellmyer and M.J. O'Shea, J. Less-Common Metals 9459 (1983).

[59] R.W. Cochrane, R. Harris and M.J. Zuckermann, Phys. Reports 481 (1979).

[60] Chapter 6 in ref 1 , and references therein.

[61] B. Boucher, A. Llénard, J.P. Rebouillat and J. Schweizer, J. Phys. F9 1421 (1979).

[62] A. Aharony and E. Pytte, Phys. Rev. Lett. 451583 (1980).

[63] J.B. Bieri, J, Sanchez, A. Fert, D. Bertrand and A.R. Fert, J. App1 Phys. 53 234? (1982).

[64] G. Czjzek, Nucl. Inst. and Meth. 199.37 (1982); Hyperfine Interactions 14189 (1983).

[65] J.M. Friedt, T. Maurer, J.P. Sanchez and J. Durand, J. Phys. E12 821 (1982).

[66] G. Czjzek, D. Weschenfelder, V. Oesterich, H. Schmidt, A. Vaures and M. Maures, J. Noncryst. Solids 61-62-433 (1984).

[67] B.D. Rainford, V. Samadian, R.J. Begum, E. T. Lee and S.K. Burke, J. App1. Phys. 537725 (1982).

[68] G. Czjzek, J. Fink, F. Götz, M. Schmidt, J.M.D. Coey, J.P. Reboufllat and A. Liénard, Phys. Rev. B23 2513 (1981).

(69] E. Borchi, S, de Gennaro, J, Phys, F $1 \overline{1147}$ (1981).

[70] E.J. Litterst, J. Physique, 36 L197 (1975).

[71] J.M.D. Coey, E. Devlin and R.J. Gambino, J. Appl. Phys. 53 $7810(1982)$.

[72] Chapter $V$ in reference 1 , and references therein.

[73] W. Nagele, R. Knorr, W. Prandl, P. Convert and J.L. Bueroz, J. Phys. C11 3295 (1978).

[74] M. Ayachi and J. Ferrf, Phys. Rev. Lett. 50 27A

[75] J.M.D. Coey and P.J.K. Murphy, J. Noncryst. Solids 50125 (1982).

[76] G. Ferey, F. Varret \& J.M.D. Coey, J. Phys. C12 L531 (1979)

[77] E.M. Gyorgy, K. Nassau, M. Eibschutz, J.V. Waezczak, C.A. Wang and J.C. Shelton, J. Appl. Phys, 502883 (1979).

(78) T. Kaneko, K. Shirakawa, S. Ohnuma, M. Nose, H. Fujimori and T. Masumoto, J. App1. Phys. 521826 (1981). 